\title{
Change, challenge, and a farewell
}

\author{
Debra Patt, MD, MBA, MPH
}

The only thing that is constant is change. - Heraclitus

W e are in a time of transition in oncology. During this period of remarkable innovation in oncology treatments we have substantial improvements in cancer care, but we also have higher health care costs and a growing population of older patients who have a disproportionate risk of developing cancer. Our successes are real, with a 1.5\% reduction in cancer mortality in the United States every year for the last decade and a growing population of cancer survivors. ${ }^{1}$ These successes are due to novel research, improved screening, early detection, and better therapeutic intervention. It is estimated that the United States spent $\$ 173$ billion on cancer care last year. While the Medicare population is expected to grow by $50 \%$ in the next 15 years (from 54 million beneficiaries in 2015 to 80 million in 2030), costs will continue to rise and we will continue to face the challenge of how to pay for the health care of this growing population. Several pilot experiments have been introduced recently to either just reduce cost or enhance value.

\section{CMS's ASP experiment}

In early March, the Centers for Medicare and Medicaid Services announced a mandatory pilot plan to test whether changes in the reimbursements for Part B prescription drugs could reduce costs and maintain or improve the quality of care. Medicare beneficiaries will be divided by zip code into the study and control groups. Phase 1 of the Part B Drug Payment Model will be implemented in the fall and will reduce the current Part $B$ reimbursement of average sales price (ASP) plus $6 \%$ to ASP plus $2.5 \%$ plus a flat fee of $\$ 16.80$ (for the study group). The second phase, likely to start in 2017, would be applied to larger proportion of the Medicare population that would be divided into reimbursement cohorts by percentage of ASP, flat fee, valueor incentive-based pricing, and absence of copays. It was surprising to see this blunt instrument policy put forth by CMS without input from the stakeholders, especially when the natural consequence of this policy intervention could result in diminished access to care for Medicare beneficiaries and possible shifts to more expensive sites of care.

\section{The oncology care model}

At the end of March, oncology practices were notified about whether they would be participating in the CMMI Institution's alternative payment model, the oncology care model. The goal of this model is to improve the quality of cancer care for Medicare beneficiaries and to incentivize value-based management of patients. There would have to be significant system changes for this model is to be put into operation in oncology. If practices are not ready, they will need to develop ways to meet these new expectations, comply with the Institute of Medicine's quality measures, facilitate compliance with evidence-based guidelines, and reduce the cost of care where appropriate.

\section{The Triple Aim}

There is much discussion about the Institute for Healthcare Improvement's Triple Aim model that focuses on better health care outcomes for the population, improved patient satisfaction with care, and lower cost per capita. Although we will all work toward those goals, the outcomes of these policy interventions have yet to be studied. We will need to evaluate the outcomes of these interventions to fully understand their impact on efficacy of treatment, reduction of toxicities, patient satisfaction, health literacy, patient quality of life, and cost of care. To do this, we will need to aggregate information from the oncology care model and determine if this well thought out plan will be effective in achieving the triple aim.

In line with these changes, I will be stepping away from The Journal of Community and Supportive ONCOLOGY to work more closely with clinical informatics. I hope that this endeavor will shed light on how we use data systems to measure and improve the delivery of cancer care. It has been my great pleasure to serve JCSO and its readers these last several years together with the talented staff and thought leaders who are my colleagues on the editorial board. Your work here makes community and supportive oncology better, and I am grateful for having had the opportunity to work closely with each of you. We have much work ahead of us.

With great appreciation,

Debra Patt

JCSO 2016;14:137. (02016 Frontline Medical Communications. doi: 10.12788/jcso.0251. 